\title{
El investigador como topo
}

\author{
Reseña del libro de \\ María del Carmen Castro Vásquez:
}

\author{
En letras chiquitas. \\ Construcción de ciudadanía y el derecho a \\ la información en salud $(2016)^{1}$
}

\author{
Maren Von der Borch*
}

Conozco a la autora desde hace más de tres décadas, ya que fuimos compañeras de trabajo en varias ocasiones. A lo largo de estos años, he observado cómo ha ido forjando su camino hasta llegar a ser la investigadora de altos vuelos que es ahora, reconocida como tal por el propio CONACYT.

Carmen Castro procedió exactamente como E.P. Thompson dijo que debe proceder un historiador: como topo que emerge a la superficie y -medio cegatón al principio- explora la pequeña franja alrededor del agujero que había cavado; luego, trazando círculos cada vez más grandes, va detectando porciones cada vez más amplias del terreno alrededor del agujero, hasta que finalmente puede descubrir toda el área que le interesa.

Así, Carmen empezó interesándose en las dimensiones socioculturales de la problemática en torno al cáncer cervicouterino $(\mathrm{CaCu})$, extendiendo su interés al cáncer mamario (siendo estos dos los cánceres que tienen que ver con la sexualidad de las mujeres y en torno a las cuales se han ido construyendo una serie de mitos, tabúes y prohibiciones). Luego, fue trazando círculos cada vez más grandes -tanto en su trabajo empírico como en la construcción de perspectivas teóricas- centrando la mirada sucesivamente en los problemas de la detección oportuna, la salud reproductiva en general, el campo médico (en sentido de Bourdieu) y la calidad de la atención en salud, los derechos de los pacientes y las posibilidades de la participación social en los programas de prevención y atención, las implicaciones de la desigualdad de género en la atención en salud, y finalmente, trazó el último círculo más amplio: la construcción de ciudadanía; es decir, la conversión de pacientes en exigentes (en palabras de ella misma) $)^{2}$ o de pacientes en agentes (en palabras de Amartya Sen). ${ }^{3}$

Hay que resaltar aquí que al parecer, el interés de la autora no está en señalar las grandes injusticias o errores médicos que

\footnotetext{
* Socióloga. MTC del Departamento de Trabajo Social y profesora de la Maestría en Políticas y Gestión del Desarrollo Social, de la Universidad de Sonora, marenv@sociales.uson.mx

${ }^{1}$ Hermosillo, Sonora: El Colegio de Sonora. Esta reseña es la versión ligeramente abreviada del texto leído el 3 de febrero de 2017 en la plaza Hidalgo, en el marco del 35 Aniversario de El Colegio de Sonora.

${ }^{2}$ Castro Vásquez, María del Carmen. (2008). De pacientes a exigentes. Un estudio sociológico sobre la calidad de la atención, derechos y ciudadanía en salud. Hermosillo, Sonora: El Colegio de Sonora.

${ }^{3}$ Sen, Amartya K. (2000). Desarrollo y libertad. Barcelona: Planeta.
} 
suelen arreglarse en la Corte. No se trata aquí de promover una sociedad en donde ser exigentes se traduce en demandas constantes contra los hospitales o el personal médico.

El propósito de Carmen Castro, claramente, es otro: se trata de aportar elementos para que particularmente las mujeres, cuyas vidas transcurren por cauces precarias, puedan participar de manera informada, crítica y responsable en las decisiones que afectan sus vidas, y en particular, que afectan sus procesos de prevención/atención en salud.

Con esto, la autora inserta su obra en ese novedoso tema de investigación que suele aparecer bajo el rótulo de democracia cotidiana: los miles de pequeños procesos de negociación que a diario ocurren en los hospitales y consultorios médicos; y que por la mala distribución de los conocimientos y la asimetría en las relaciones de poder entre pacientes (especialmente mujeres en situación precaria) y personal médico, tienden a profundizar las desigualdades sociales preexistentes.

En letras chiquitas... es la culminación de esta paciente labor de Carmen Castro realizada a través de años. El punto de partida es la idea de que los derechos de las mujeres están claramente definidos y reglamentados, pero por una serie de causas complejas, el ejercicio efectivo de estos derechos por parte de las mujeres, está totalmente rezagado.

La norma oficial mexicana NOM-014 (que se ocupa de la "prevención, detección, diagnóstico, tratamiento, control y vigilancia epidemiológica del cáncer cervicouterino" [:58]), por ejemplo, estipula que las mujeres tienen derecho a conocer la prueba del PAP; recibir información sobre los tipos de cáncer y sus factores de riesgo, así como conocer los resultados (:58). Pero -como se puede ver en los testimonios de las mujeres reunidos en el libro- rara vez se ejerce este derecho de manera efectiva.

El material primario de este libro lo constituyen las entrevistas extensamente citadas (lo que es uno de los mayores aciertos de la autora), obtenidas a través de 14 grupos focales $(\mathrm{GF})$ que se conformaron según el criterio de si las mujeres estaban organizadas o no - organizadas; por ejemplo, como beneficiarias del Programa Oportunidades (hoy Prospera), como grupo comunitario de base dedicado a la gestión de servicios, o como organización feminista o de lucha por los derechos sexuales y reproductivos. El planteamiento fue:

Saber si la participación de las mujeres en grupos organizados aportaba a su apropiación de derechos y a sus prácticas en la atención de su salud, así como a un ejercicio de derechos... $(: 56)$.

El segundo criterio era la edad: algunos de los GF convocaron a las más jóvenes (de entre 25 y 35 años de edad) y otros a las mayores (de entre 36 a 45 años). Todas eran usuarias de los servicios públicos en salud, y todas radicaban en la periferia de Hermosillo o en Estación Pesqueira (un poblado formado por jornaleros migrantes en su mayoría, en torno a las necesidades de mano de obra de los campos agrícolas de la zona, dedicados a la producción de uva de mesa); y todas

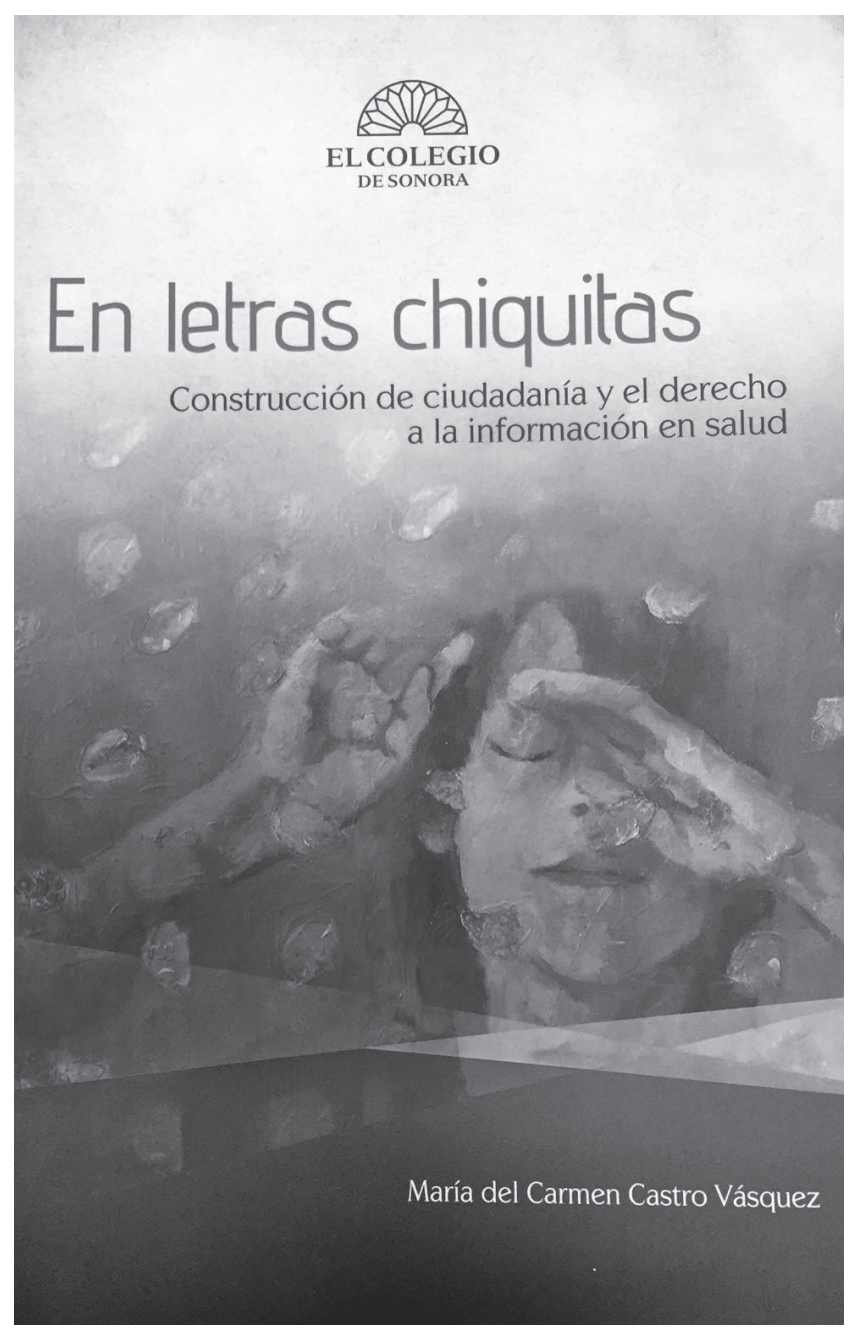

Se habían sometido a la prueba del PAP cuando menos una vez en los últimos tres años, al momento de formar el GF. Todas estaban al cuidado de la salud de hijos pequeños o adolescentes y jóvenes, y acudían más o menos de manera regular a los programas de detección oportuna del cáncer (:57).

En total fueron 107 mujeres las que participaron en los diferentes grupos focales - de ahí la gran riqueza de experiencias, subjetividades, prácticas y estrategias vertidas en este libro. Pero la autora no se conformó con este material. Consciente del principio de que "La narrativa del GF [...] se construye frente al 'otro', y sus participantes en lo general se suman al discurso construido entre todas [...] aunque se guarden las particularidades individuales" (:55), quería confrontar ese material con información "desde dentro" (:55). Con esta idea en mente, ella y su equipo de colaboradoras realizaron 12 entrevistas a profundidad (individuales), cada una de las cuales contenía más de 100 preguntas.

La perspectiva teórica (o marco teórico) de la investigación, se construyó a partir de una impresionante -por su amplitud y profundidad- labor de revisión de conceptos, teorías y paradigmas - arribando finalmente a la decisión de privilegiar dos abordajes complementarios entre sí: 
1. La perspectiva basada en conceptos como ciudadanía, derechos (especialmente el derecho a la información), empoderamiento, agencia, participación social o comunitaria todos ellos vistos desde el mirador de la desigualdad social y la desigualdad de género; $y$

2. La teoría de la práctica de Pierre Bourdieu, que parte de conceptos como hábitus (en especial el hábitus lingüístico), campo (campo médico, en este caso), dominación y violencia simbólica. El concepto central para la autora es el de hábitus, definida por ella como condición que no es natural, sino que ha sido socialmente construida, y que se "refiere a las capacidades de todas las personas para manejarse en la sociedad; representa el cúmulo de aprendizajes internalizados desde que se vive en sociedad; y es el esquema que da estructura a las prácticas y percepciones" (:145).

La problemática general en torno a la cual gira este libro, es (como ya se mencionó anteriormente) el desfase aparente entre los derechos a la salud -y en especial el derecho a la información- plasmados en la normatividad mexicana, por un lado; y las prácticas y estrategias puestas en marcha en el ejercicio de estos derechos, por el otro. Esta problemática general fue desagregada en cuatro vertientes principales, por ejemplo, las siguientes:

1. El derecho a la salud tal como aparece en el discurso de las mujeres (cap. III);

2. La experiencia de la participación de las mujeres en el Programa de Oportunidades (lo que la autora llama "participar sin participar"; cap. IV);

3. Las prácticas y estrategias de las mujeres puestas en juego para apropiarse o no, de sus derechos (cap. V); y finalmente,

4. La existencia -o más bien el enfrentamiento- de dos lenguajes (o dos hábitos lingüísticos) en el campo de la salud: el lenguaje técnico del personal médico y el lenguaje lego de las usuarias de los servicios de salud. Un acierto de la autora es que ella ve esto no solo como un problema de comunicación, o de dificultad en el acceso a la información, sino como "forma de operar de un mecanismo de subordinación de las mujeres, al reconocerse en desventaja frente al lenguaje técnico de su interlocutor" (:175).

Al resumen de los hallazgos principales que la autora hace al final de su libro, solo quiero agregar una cosa que me llamó mucho la atención: a pesar de que todas las mujeres tienen nociones más o menos claras de que son poseedoras de derechos, en su intento por definir estos derechos prácticamente ninguna se refiere a la Constitución Mexicana, a la Declaración Universal (1948) o a los tratados internacionales firmados por México (los cuales, según lo que estipula nuestra Carta Magna, deben ser respetados igual que el resto de lo que establece la Constitución).

Pienso que este desconocimiento es el principal responsable de las ciudadanías de tercera, cuarta o quinta que viven especialmente las mujeres de condiciones de vida precarias, ya que las prácticas y estrategias que ponen en marcha para resolver sus problemas -tal y como aparecen en los testimonios reunidos en este libro- no se guían por una visión de derechos, sino por la lógica de la beneficencia, de la ayuda o dádiva que el gobierno les da a los pobres. Esta lógica, como todos sabemos, embona muy bien con la tendencia actual de desmantelar lo que queda del Estado de Bienestar.

Un comentario final, con el título que puso a su libro ( $E n$ letras chiquitas...), la autora se revela como buena lectora de literatura policiaca: desde el principio, nos deja en suspenso sobre su significado. Obviamente, no voy a correr la cortina aquí: no quiero estropearles el placer que seguramente experimentarán al acercarse a una lectura cuidadosa de este texto tan necesario.

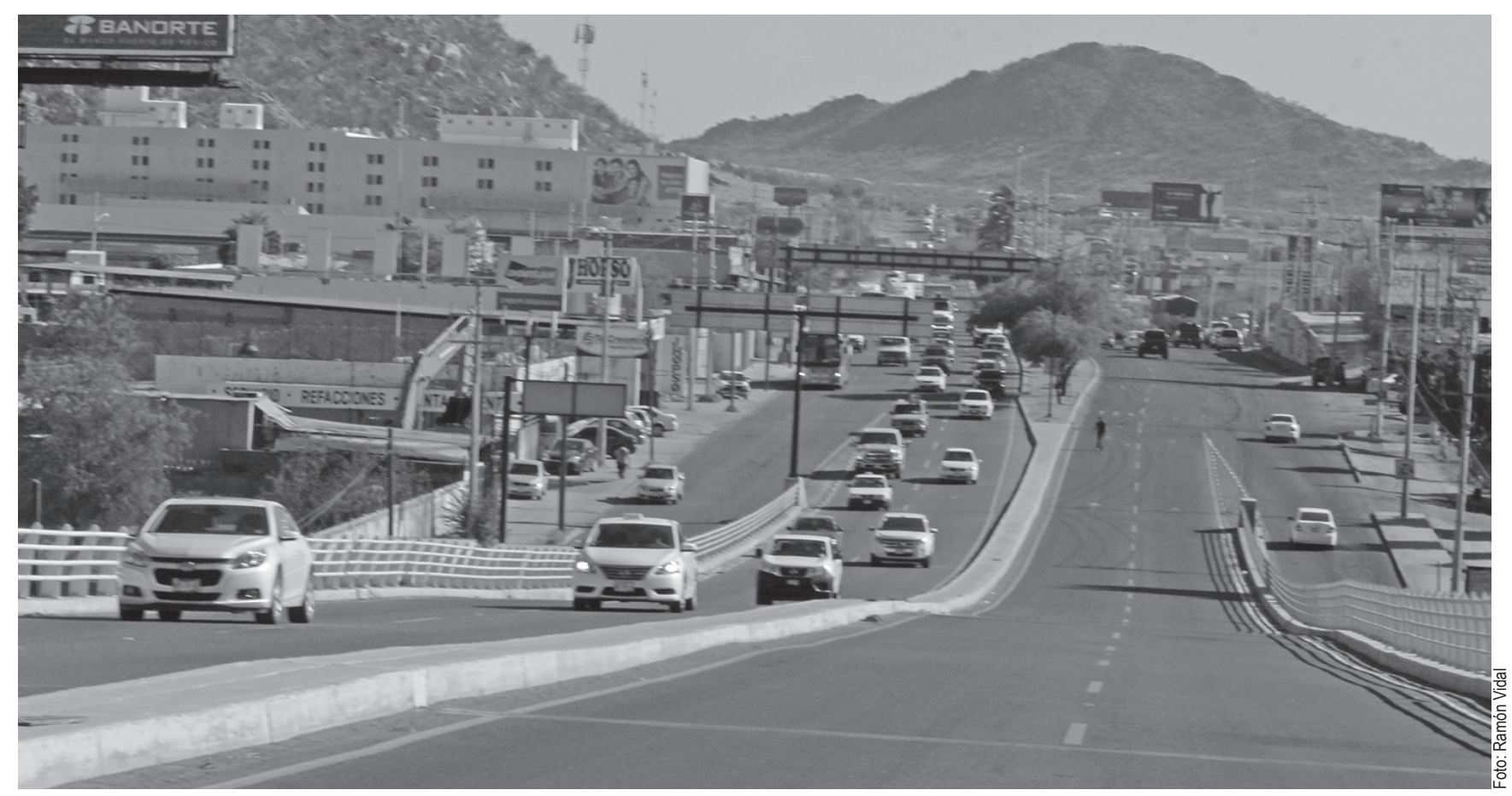

